\title{
A magnetic levitation based low-gravity simulator with an unprecedented large functional volume
}

\author{
Hamid Sanavandi $\mathbb{D}^{1,2}$ and Wei Guo $\mathbb{i D}^{1,2 凶}$
}

Low-gravity environment can have a profound impact on the behaviors of biological systems, the dynamics of fluids, and the growth of materials. Systematic research on the effects of gravity is crucial for advancing our knowledge and for the success of space missions. Due to the high cost and the limitations in the payload size and mass in typical spaceflight missions, ground-based low-gravity simulators have become indispensable for preparing spaceflight experiments and for serving as stand-alone research platforms. Among various simulator systems, the magnetic levitation-based simulator (MLS) has received long-lasting interest due to its easily adjustable gravity and practically unlimited operation time. However, a recognized issue with MLSs is their highly nonuniform force field. For a solenoid MLS, the functional volume $V_{1 \%}$, where the net force results in an acceleration $<1 \%$ of the Earth's gravity $g$, is typically a few microliters $(\mu \mathrm{L})$ or less. In this work, we report an innovative MLS design that integrates a superconducting magnet with a gradient-field Maxwell coil. Through an optimization analysis, we show that an unprecedented $V_{1 \%}$ of over $4000 \mu \mathrm{L}$ can be achieved in a compact coil with a diameter of $8 \mathrm{~cm}$. We also discuss how such an MLS can be made using existing high- $T_{c}$-superconducting materials. When the current in this MLS is reduced to emulate the gravity on Mars $\left(g_{M}=0.38 g\right)$, a functional volume where the gravity varies within a few percent of $g_{M}$ can exceed $20,000 \mu \mathrm{L}$. Our design may break new ground for future low-gravity research.

npj Microgravity (2021)7:40; https://doi.org/10.1038/s41526-021-00174-4

\section{INTRODUCTION}

Reduced gravity is known to have important effects on various biological and physical systems. For instance, a weightless environment may prohibit cell culture growth ${ }^{1}$ and may cause cellular stressors and bone loss that can negatively impact astronauts' health ${ }^{2-4}$. In fluid systems, reduced gravity can significantly affect the sloshing dynamics of cryogenic propellants in spacecrafts ${ }^{5}$, the surface oscillation of liquid drops ${ }^{6}$, bubble cavitation $^{7}$, and boiling heat transfer in fluids ${ }^{8,9}$. In material science, the potential of reduced gravity in growing tissues ${ }^{10}$ and crystals $^{11}$ and for materials processing ${ }^{12}$ has been recognized. Conducting systematic research to understand the mechanism of gravity in these diverse systems will undoubtedly advance our knowledge. Furthermore, various programs initiated recently by public space agencies and private organizations ${ }^{13-15}$ aiming at long-term human habitation of the Moon and Mars have further strengthened the needs of experimental research in low-gravity environments.

The ideal microgravity condition can be achieved in spaceflight experiments conducted during space-shuttle missions ${ }^{16}$ and at space stations ${ }^{17}$. However, these experiments are limited by the high cost and the small payload size and mass ${ }^{18}$. The fact that the astronauts have to conduct the experiments instead of the trained scientists also put constraints on the design of the experiments. For these reasons, researchers have devoted great efforts to developing ground-based low-gravity simulators. One major category, which utilizes free fall to generate near-zero gravity, includes drop towers ${ }^{19,20}$, parabolic aircraft ${ }^{21,22}$, sounding rockets $^{23}$, and suborbital rocketry ${ }^{24}$. Despite their usefulness, a known limitation of these facilities is the relatively short low-gravity duration (i.e., from several seconds to a few minutes ${ }^{25}$ ), which makes them unsuitable for experiments that require long observation times ${ }^{26}$. In biological and medical research, rotational facilities such as clinostat machines ${ }^{27,28}$, rotating wall vessels ${ }^{29}$, and random positioning machines ${ }^{30}$ are also adopted to achieve a small time-averaged gravity vector ${ }^{31,32}$. Although these simulators are convenient, they do not produce a genuine low-gravity environment and can generate unwanted centrifugal forces and circulating flows in the samples ${ }^{31-33}$.

On the other hand, magnetic field-gradient levitation of various diamagnetic materials has been demonstrated ${ }^{34-36}$. Even living organisms have been successfully levitated ${ }^{37-41}$, and there is no evidence of any cumulative harmful effects due to the field exposure ${ }^{40-42}$. Compared to other low-gravity simulator systems, a magnetic levitation-based simulator (MLS) offers unique advantages, including low cost, easy accessibility, adjustable gravity, and practically unlimited operation time $\mathrm{s}^{37,38,43}$. However, a known issue with MLSs is their highly non-uniform force field around the levitation point. If we define a $0.01-g$ functional volume $V_{1 \%}$ where the net force results in an acceleration less than $1 \%$ of the Earth's gravity $g, V_{1 \%}$ is typically less than a few microlitres $(\mu L)$ for conventional solenoid MLSs. Although diamagnetic samples with sizes larger than $V_{1 \%}$ can be levitated, a stress field caused by the residue force inside the samples can compromise the measurement results. Despite some limited efforts in designing MLSs for improved functional volumes ${ }^{44-46}$, major progress is still lacking. Furthermore, the high energy consumption rate of conventional resistive solenoid MLSs is also concerning. For instance, $4 \mathrm{MW}$ electric power is required to levitate a frog using a resistive solenoid $\mathrm{MLS}^{37}$.

In this paper, we report an innovative MLS design that consists of a gradient-field Maxwell coil placed in the bore of a superconducting (SC) magnet. By optimizing the SC magnet's field strength and the current in the Maxwell coil, we show that an

${ }^{1}$ National High Magnetic Field Laboratory, 1800 East Paul Dirac Drive, Tallahassee, FL 32310, USA. ${ }^{2}$ Mechanical Engineering Department, FAMU-FSU College of Engineering, Florida State University, Tallahassee, FL 32310, USA. ${ }^{凶}$ email: wguo@magnet.fsu.edu 
unprecedented $V_{1 \%}$ of over $4000 \mu \mathrm{L}$ can be achieved in a compact coil of $8 \mathrm{~cm}$ in diameter. This optimum $V_{1 \%}$ increases with the size and the field strength of the MLS. We then discuss how such an MLS can be made using existing high- $T_{c} S C$ materials so that longtime operation with minimal energy consumption can be achieved. To further demonstrate the usefulness of this MLS, we also consider reducing its current and the field strength to emulate the gravity on Mars $\left(g_{M}=0.38 \mathrm{~g}\right)$. It turns out that a functional volume over $20,000 \mu \mathrm{L}$ can be produced, in which the gravity only varies within a few percent of $g_{M}$. Our design concept may break new ground for exciting applications of MLSs in future low-gravity research.

\section{RESULTS}

\section{Levitation by a solenoid magnet}

To aid the discussion of our MLS design, we first introduce the fundamentals of magnetic levitation using a solenoid magnet. Following this discussion, we will present the details of our innovative MLS design concept.

The mechanism of magnetic levitation can be understood by considering a small sample (volume $\Delta V$ ) placed in a static magnetic field $\mathbf{B}(\mathbf{r})$. Owing to the magnetization of the sample material, the energy of the magnetic field increases by ${ }^{47}$

$$
\Delta E_{B}=\frac{-\chi B^{2}(\mathbf{r})}{2 \mu_{0}(1+\chi)} \Delta V
$$

where $X$ is the magnetic susceptibility of the sample material and $\mu_{0}$ is the vacuum permeability. For diamagnetic materials with a negative $X, \Delta E_{B}$ is positive and therefore it requires energy to insert a diamagnetic sample into the $\mathbf{B}(\mathbf{r})$ field. Counting in the gravity effect, the total potential energy associated with the sample per unit volume can be written as:

$$
E(\mathbf{r})=\frac{-\chi B^{2}(\mathbf{r})}{2 \mu_{0}(1+\chi)}+\rho g z
$$

where $\rho$ is the material density and $z$ denotes the vertical coordinate. This energy leads to a force per unit volume acting on the sample as:

$$
\mathbf{F}=-\nabla E(\mathbf{r})=\frac{X}{\mu_{0}(1+\chi)}(\nabla \mathbf{B}) \cdot \mathbf{B}-\rho g \hat{e}_{z} .
$$

For an appropriate non-uniform magnetic field, the vertical component of the field-gradient force (i.e., the first term on the right side in Eq. (3)) may balance the gravitational force at a particular location, i.e., the levitation point. Sample suspension can therefore be achieved at this point.

In order to attain a stable levitation, the specific potential energy $E$ must have a local minimum at the levitation point so the sample cannot stray away. Since $E$ depends on the material properties besides the $\mathbf{B}(\mathbf{r})$ field, we need to specify the sample material. Considering the fact that water has been utilized in a wide range of low-gravity researches ${ }^{48-50}$ and is also the main constituent of living cells and organisms ${ }^{51}$, we adopt the water properties at ambient temperature ${ }^{52}$ (i.e., $X=-9.1 \times 10^{-6}$ and $\rho=$ $10^{3} \mathrm{~kg} / \mathrm{m}^{3}$ ) in all subsequent analyses. To see the effect of the $\mathbf{B}(\mathbf{r})$ field, we consider a solenoid with a diameter of $D=8 \mathrm{~cm}$ and a height of $\sqrt{3} D / 2$, as shown in Fig. 1a. These dimensions are chosen to match the size of the MLS that we will discuss in later sections. For a solenoid with $N$ turns and with an applied current $I$, $\mathbf{B}(\mathbf{r})$ can be calculated using a known integral formula that depends on the product $N I$ (see details in the Method section). $E(\mathbf{r})$ in the full space can then be determined.

In Fig. $1 \mathrm{~b}$, we show the calculated $E(\mathbf{r})$ near the top opening of the solenoid when a turn-current of $\mathrm{NI}=607.5 \mathrm{kA}$ is applied. In general, $E$ is high near the solenoid wall due to the strong $\mathbf{B}$ field there. Slightly above the solenoid, there is a trapping region
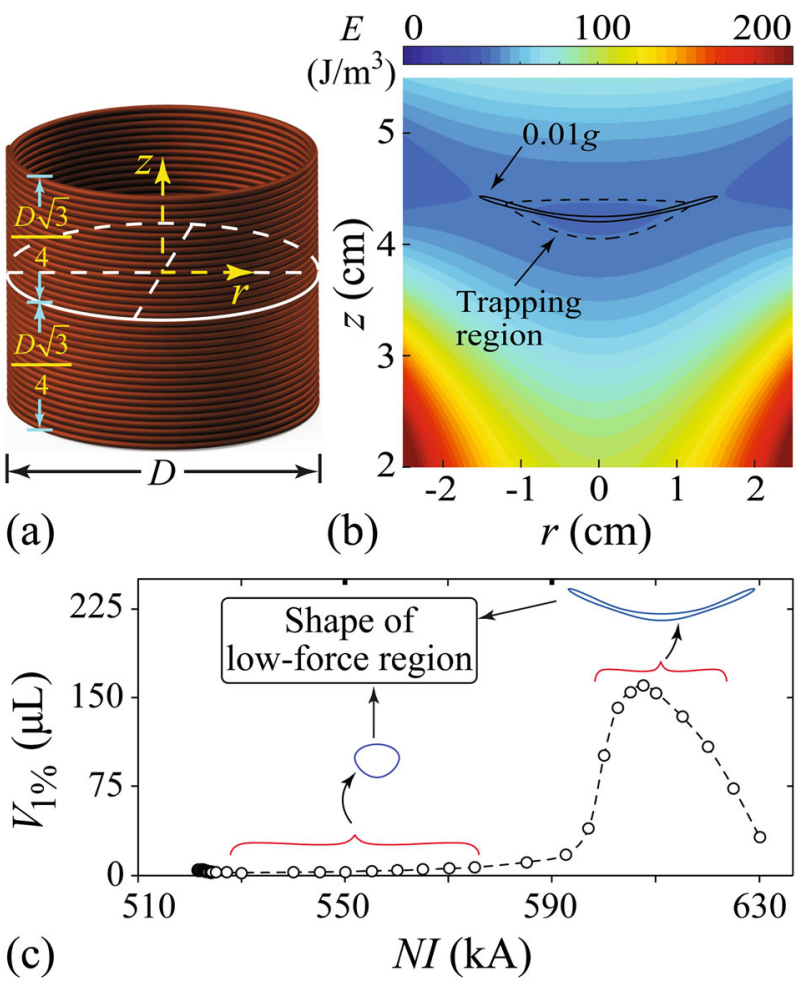

Fig. 1 Functional volume analysis for a conventional solenoid MLS. a Schematic of a solenoid with a diameter of $D=8 \mathrm{~cm}$ and a height of $\sqrt{3} D / 2$. b Calculated specific potential energy $E(\mathbf{r})$ of a small water sample placed in the magnetic field. The turn-current $N I$ of the solenoid is $607.5 \mathrm{kA}$. The origin of the coordinates is at the center of the solenoid. The dashed contour denotes the boundary of the trapping region, and the solid contour shows the low-force region (i.e., acceleration $<0.01 \mathrm{~g}$ ). c The functional volume $V_{1 \%}$ (i.e., the overlapping volume of the two contours) versus the turn-current NI. Representative shapes of the low-force region are shown.

(enclosed by the dashed contour) in which $E$ decreases towards the region center. When a water sample is placed in this region, it moves towards the region center where the net force is zero, i.e., the levitation point. We have also calculated the specific force field using Eq. (3). The solid contour in Fig. 1b denotes the low-force region in which the net force corresponds to an acceleration $<0.01 \mathrm{~g}$. The overlapping volume of the trapping region and the low-force region is defined as our functional volume $V_{1 \%}$ where the sample not only experiences a weak residue force but also remains trapped. In Fig. $1 c$, we show the calculated $V_{1 \%}$ as a function of NI. The trapping region emerges only above a threshold turn-current of about $N I=520 \mathrm{kA}$. As $N I$ increases, $V_{1 \%}$ first remains small (i.e., a few $\mu \mathrm{L}$ ) and has a shape like an inverted raindrop. When $\mathrm{Nl}$ is above $\sim 600 \mathrm{kA}, V_{1 \%}$ grows rapidly and peaks at $\mathrm{NI}=607.5 \mathrm{kA}$ before it drops with further increasing $\mathrm{NI}$. In the peak regime, $V_{1 \%}$ has a highly anisotropic shape due to the nonuniform force field, which makes it unsuitable for practical applications despite the enhanced $V_{1 \%}$ value. The required extremely large turn-current also presents a great challenge.

\section{Concept and performance of our MLS design}

To increase $V_{1 \%}$, the key is to produce a more uniform fieldgradient force to balance the gravitational force such that the net force remains low in a large volume. Base on Eq. (3), this can be achieved if we have a nearly uniform B field and in the meanwhile, the field gradient is almost constant in the same volume. These two seemingly irreconcilable conditions can be satisfied approximately. The solution is to superimpose a strong uniform field $\mathbf{B}_{0}$ 


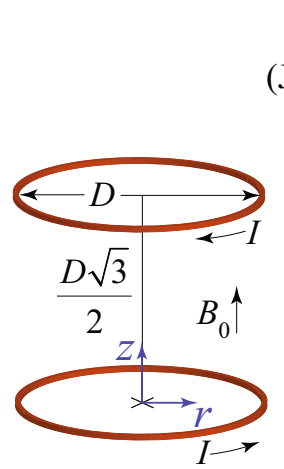

(a)

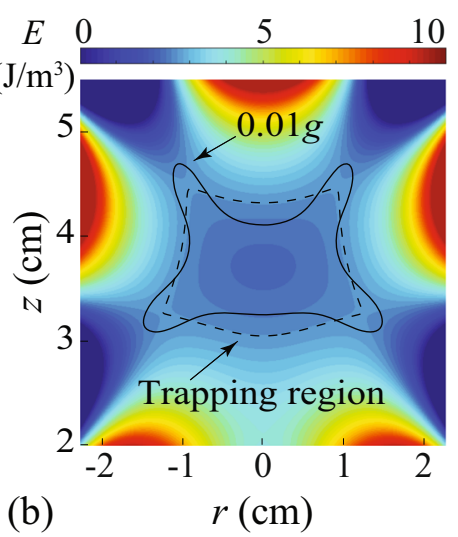

(b)
Fig. 2 Functional volume of our MLS design using a gradient-field Maxwell coil. a Schematic of the gradient-field Maxwell coil with a diameter $D=8 \mathrm{~cm}$ in the presence of an applied uniform field $\mathbf{B}_{0} . \mathbf{b}$ Calculated specific potential energy $E(\mathbf{r})$ of a small water sample placed in the magnetic field for $I=112.6 \mathrm{kA}$ and $B_{0}=24 \mathrm{~T}$. The origin of the coordinates is at the center of the bottom current loop. The black dashed contour denotes the boundary of the trapping region, and the black solid contour shows the low-force region (i.e., acceleration $<0.01 \mathrm{~g}$ ).

with a weak field $\mathbf{B}_{1}(\mathbf{r})$ that has a fairly constant field-gradient $\nabla \mathbf{B}_{1}$. In this way, the total field $\mathbf{B}=\mathbf{B}_{0}+\mathbf{B}_{1} \simeq \mathbf{B}_{0}$ is approximately uniform and its gradient $\nabla \mathbf{B} \simeq \nabla \mathbf{B}_{1}$ can also remain nearly constant.

The uniform field $\mathbf{B}_{0}$ can be produced in the bore of a superconducting solenoid magnet. Indeed, for superconducting magnets used in magnetic resonance imaging applications, spatial uniformity of the field better than a few parts per million (ppm) in a space large enough to hold a person has become standard ${ }^{53-55}$. The recently built 32-T all-superconducting magnet at the National High Magnetic Field Laboratory (NHMFL) further proves the feasibility of producing strong uniform fields using cutting-edge superconducting technology ${ }^{56}$. As for the $\mathbf{B}_{1}$ field, we propose to produce it using a gradient-field Maxwell coil ${ }^{57}$. As shown in Fig. 2a, such a coil consists of two identical current loops (diameter $D$ ) placed coaxially at a separation distance of $\sqrt{3} D / 2$. The current in the top loop is clockwise (viewed from the top) while the current in the bottom loop is counterclockwise. It was first demonstrated by Maxwell that such a coil configuration could produce a highly uniform field gradient in the region between the two loops ${ }^{57}$.

The $\mathbf{B}_{1}(\mathbf{r})$ generated by the gradient-field Maxwell coil can be calculated using the Biot-Savart law ${ }^{47}$ (see details in the Method section), from which the specific potential energy $E$ for an inserted water sample can again be determined. As an example, we show in Fig. $2 \mathrm{~b}$ the calculated $E(\mathbf{r})$ profile for a coil with $D=8 \mathrm{~cm}$ and with an applied current of $I=112.6 \mathrm{kA}$ in the presence of a uniform field $B_{0}=24 \mathrm{~T}$. Again, we use the dashed contour and the solid contour to show, respectively, the trapping region and the $0.01 \mathrm{~g}$ low-force region. By evaluating the overlapping volume of the two regions, we obtain $V_{1 \%}=4004 \mu \mathrm{L}$. More importantly, this functional volume is much more isotropic as compared with that in Fig. 1b, which makes it highly desirable in practical applications.

To optimize the coil current $I$ and the base field $B_{0}$, we have conducted further analyses. First, for a fixed $B_{0}$, we vary the coil current $I$. Representative results at $B_{0}=24 \mathrm{~T}$ are shown in Fig. 3a. It is clear that $V_{1 \%}$ peaks at about $I=112.6$. We denote this peak value as $V_{\text {opt }}$. The decrease of $V_{1 \%}$ at large $I$ is caused by the fact that the field $\mathbf{B}_{1}$ generated by the coil is no longer much smaller than the base field $\mathbf{B}_{0}$, which impairs the uniformity of the fieldgradient force. Next, we vary the base field strength $B_{0}$ and determine the corresponding $V_{\text {opt }}$ at each $B_{0}$. The result is shown
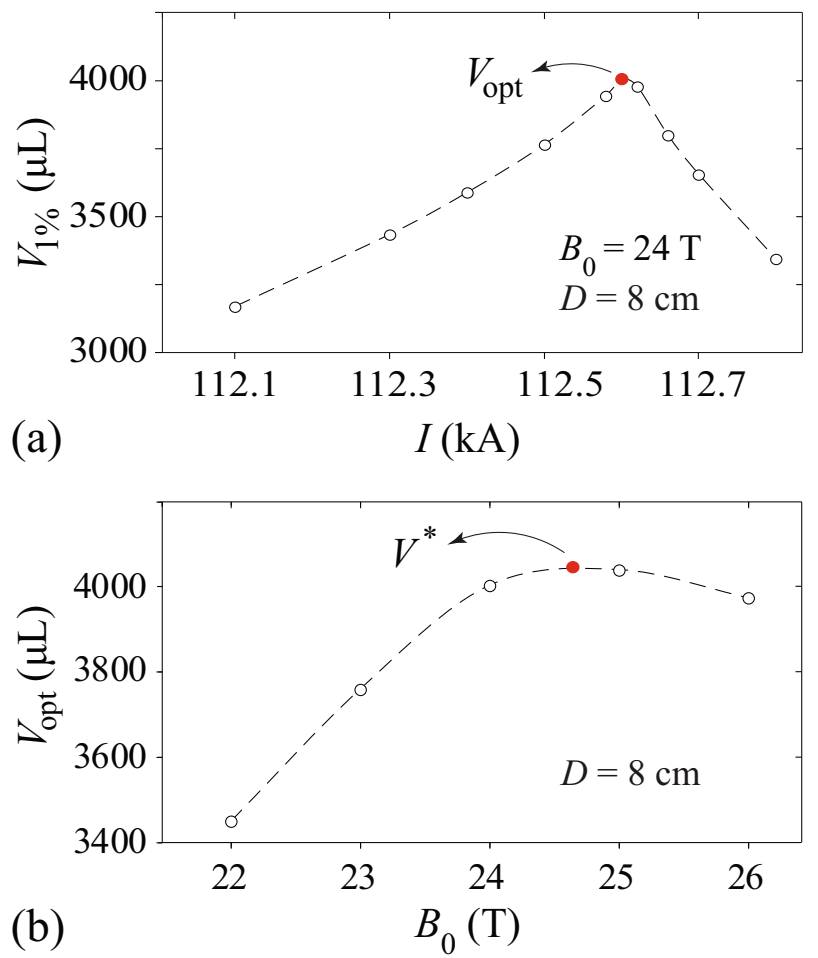

Fig. 3 Optimization analysis of the functional volume of our MLS design. a Calculated $V_{1 \%}$ versus the loop current I for the coil shown in Fig. 2 with $B_{0}=24 \mathrm{~T}$. The largest $V_{1 \%}$ is denoted as $V_{\text {opt }}$. b The obtained $V_{\text {opt }}$ as a function of $B_{0}$. The overall maximum $V_{\text {opt }}$ is denoted as $V$, and the corresponding coil current and base field are designated as $I^{*}$ and $B_{0}^{*}$, respectively.

in Fig. 3b. It turns out that there exists an optimum base field strength of $\sim 24.7 \mathrm{~T}$ (denoted as $B_{0}^{*}$ ), where an overall maximum functional volume (denoted as $V^{*}$ ) of $\sim 4050 \mu \mathrm{L}$ can be achieved. This volume is comparable to those of the largest water drops adopted in the past spaceflight experiments ${ }^{50,58}$. The above analyses assumed a fixed coil diameter $D=8 \mathrm{~cm}$. When $D$ varies, the maximum functional volume $V^{*}$ and the corresponding MLS parameters (i.e., $I^{*}$ and $B_{0}^{*}$ ) should also change. To examine the coil-size effect, we have repeated the aforementioned analyses with a number of coil diameters. The results are collected in Fig. 4. As $D$ increases from $6 \mathrm{~cm}$ to $14 \mathrm{~cm}$, the maximum functional volume $V^{*}$ increases from $\sim 1500 \mu \mathrm{L}$ to over $21,000 \mu \mathrm{L}$, i.e., over 14 times. Meanwhile, the required coil current $I^{*}$ and the base field strength $B_{0}^{*}$ increase almost linearly with $D$ by factors of $\sim 4$ and 1.3 , respectively. This analysis suggests that it is advantageous to have a larger coil provided that the desired $I^{*}$ and $B_{0}^{*}$ can be achieved.

\section{DISCUSSION}

The MLS concept that we have presented requires an applied current of the order $10^{2} \mathrm{kA}$ in both loops of the gradient-field Maxwell coil. A natural question is whether this is practical. One may consider making the loop using a thin copper wire with $10^{3}$ turns so that a current of the order $10^{2} \mathrm{~A}$ in the wire is sufficient. However, simple estimation reveals that the Joule heating in the resistive wire can become so large that the wire could melt. To solve this issue, we propose to fabricate the Maxwell coil using REBCO (i.e., rare-earth barium copper oxide) superconducting tapes similar to those used in the work by Hahn et al. ${ }^{59}$. A schematic of the proposed MLS setup is shown in Fig. 5a. A 24-T superconducting magnet with a bore diameter of $120 \mathrm{~mm}$ (existing at the NHMFL ${ }^{60,61}$ ) is assumed for producing the $\mathbf{B}_{0}$ 
field. Four sets of gradient-field Maxwell coils made of REBCO pancake rings are placed in the bore of the superconducting magnet. Each pancake ring is made of 94 turns of the REBCO tape (width: $4 \mathrm{~mm}$; thickness: $0.043 \mathrm{~mm}$ ) so its cross-section is nearly a square (i.e., $4 \mathrm{~mm}$ by $4 \mathrm{~mm}$ ). The pancake rings are arranged along the diagonal lines of a standard gradient-field Maxwell coil and the averaged diameter of the pancake rings is $\sim 8 \mathrm{~cm}$. This coil configuration is found to produce a $\mathbf{B}_{1}$ field with minimal deviations from that of an ideal gradient-field Maxwell coil. While the superconducting magnet at the NHMFL is cooled by immersion in a liquid helium bath, the compact REBCO coils could be cooled conveniently by a $4-K$ pulse-tube cryocooler inside a shielded vacuum housing. A room-temperature center bore with a diameter as large as $6 \mathrm{~cm}$ can be used for sample

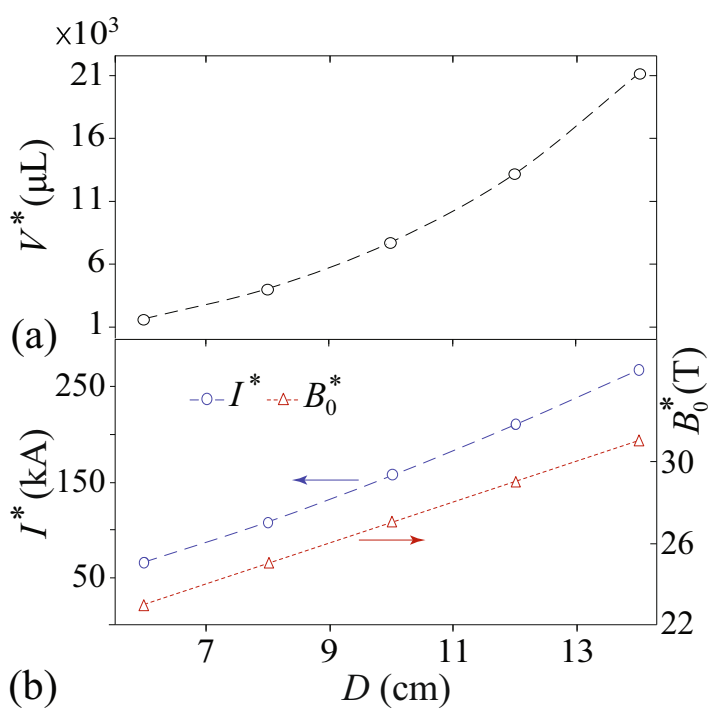

Fig. 4 Coil-size dependence of the optimal MLS parameters. a The maximum functional volume $V^{*}$ for coils with different diameters $D$. b The required optimum $I^{*}$ and $B_{0}^{*}$ to achieve $V^{*}$ versus the coil diameter $D$. loading and observation. When a current of $\sim 290 \mathrm{~A}$ is applied in the REBCO tapes, a total turn-current $N I=4 \times 94 \times 290 \mathrm{~A} \simeq 109 \mathrm{kA}$ can be achieved. Note that the quenching critical current of the REBCO tape can reach $700 \mathrm{~A}$ even under an external magnetic field of $30 \mathrm{~T}^{62}$. Therefore, operating our REBCO coils with a tape current of 290 A should be safe and reliable.

To prove the performance of the practical MLS design as depicted in Fig. 5a, we have repeated the previously presented optimization analyses. A representative plot of the specific potential energy $E(\mathbf{r})$ at a total turn-current $\mathrm{NI}=108.37 \mathrm{kA}$ and $B_{0}=24 \mathrm{~T}$ is shown in Fig. $5 \mathrm{~b}$. The overall shapes of the trapping region and the low-force region are nearly identical to those of the ideal gradient-field Maxwell coil. The dependence of $V_{1 \%}$ on the turn-current $\mathrm{NI}$ at $B_{0}=24 \mathrm{~T}$ is shown in Fig. $5 \mathrm{c}$. A peak functional volume $V_{\text {opt }}$ of $\sim 3450 \mu \mathrm{L}$ is achieved. In Fig. $5 \mathrm{~d}$, the peak volume $V_{\text {opt }}$ obtained at various base field strength $B_{0}$ is shown. Again, the trend is similar to that in Fig. 3. Therefore, despite the change in the coil geometry as compared with the ideal gradient-field Maxwell coil, the performance of our practical design does not exhibit any significant degradation.

Besides levitating samples for near-zero gravity research, our MLS can also be tuned to partially cancel the Earth's gravity so that ground-based emulation of reduced gravities in the extraterrestrial environments (such as on the Moon or Mars) can be achieved. To demonstrate this potential, we present further analyses of the practical MLS shown in Fig. 5 with lower applied currents for simulating the Martian gravity $g_{M}=0.38 g^{43}$. In Fig. $6 a$, we show a contour plot of the specific potential energy $E(\mathbf{r})$ for water samples in the practical MLS when a turn-current of $\mathrm{NI}=$ $66.55 \mathrm{kA}$ is applied at $B_{0}=24 \mathrm{~T}$. It is clear that the energy contour lines (red curves) are evenly spaced in the center region of the MLS, suggesting a fairly uniform and downward-pointing force in this region. We then calculate the magnitude of the force using Eq. (3). The two black contours in Fig. 6a represent the boundaries of the regions in which the total force leads to an effective gravitational acceleration within $1 \%$ and $5 \%$ of $g_{M}$, respectively. If we define the volume of the contour in which the gravity varies within $5 \%$ of $g_{M}$ as our functional volume $V_{M}$, its dependence on the turn-current at $B_{0}=24 \mathrm{~T}$ is shown in Fig. 6b. This functional volume has a peak value $V_{\text {opt }}$ of $\sim 22.5 \times 10^{3} \mu \mathrm{L}$ at $N I=66.55 \mathrm{kA}$.
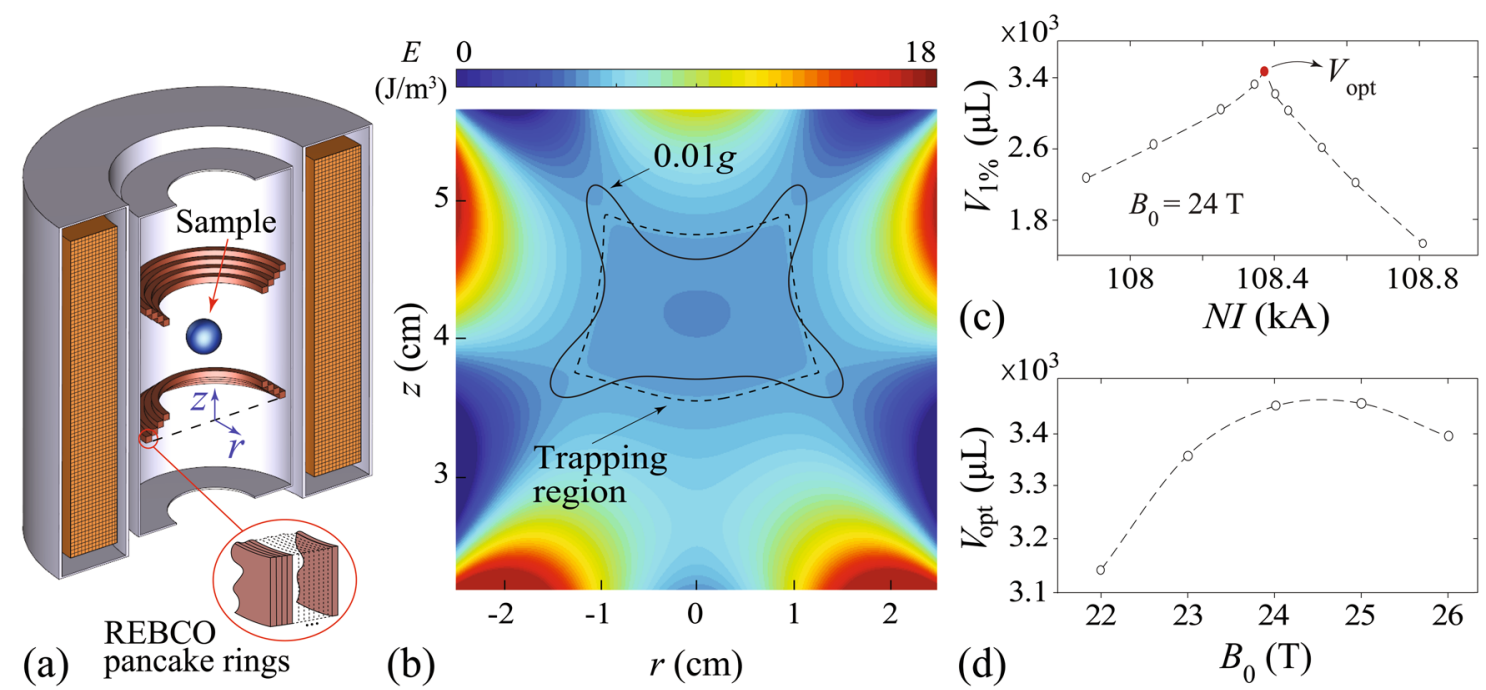

(c)

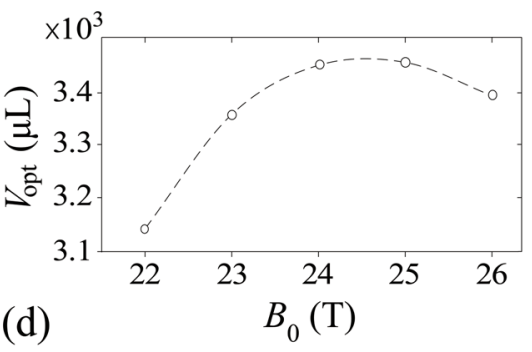

Fig. 5 Functional volume analysis for a practical MLS setup. a Schematic of a practical MLS setup that consists of a 24-T superconducting magnet with four sets of gradient-field Maxwell coils made of REBCO pancake rings. The averaged diameter of the pancake rings is $\sim 8 \mathrm{~cm}$. b Calculated specific potential energy $E(\mathbf{r})$ for a small water sample placed in this MLS with a total turn-current $N I=108.37 \mathrm{kA}$. The origin of the coordinates is at the center of the lowest pancake ring. The dashed contour denotes the boundary of the trapping region and the solid contour shows the $0.01 \mathrm{~g}$ low-force region. c Calculated $V_{1 \%}$ versus the turn-current $\mathrm{NI}$ at $B_{0}=24 \mathrm{~T}$. The peak $V_{1 \%}$ is denoted as $V_{\text {opt }}$. b The obtained $V_{\text {opt }}$ as a function of $B_{0}$. 

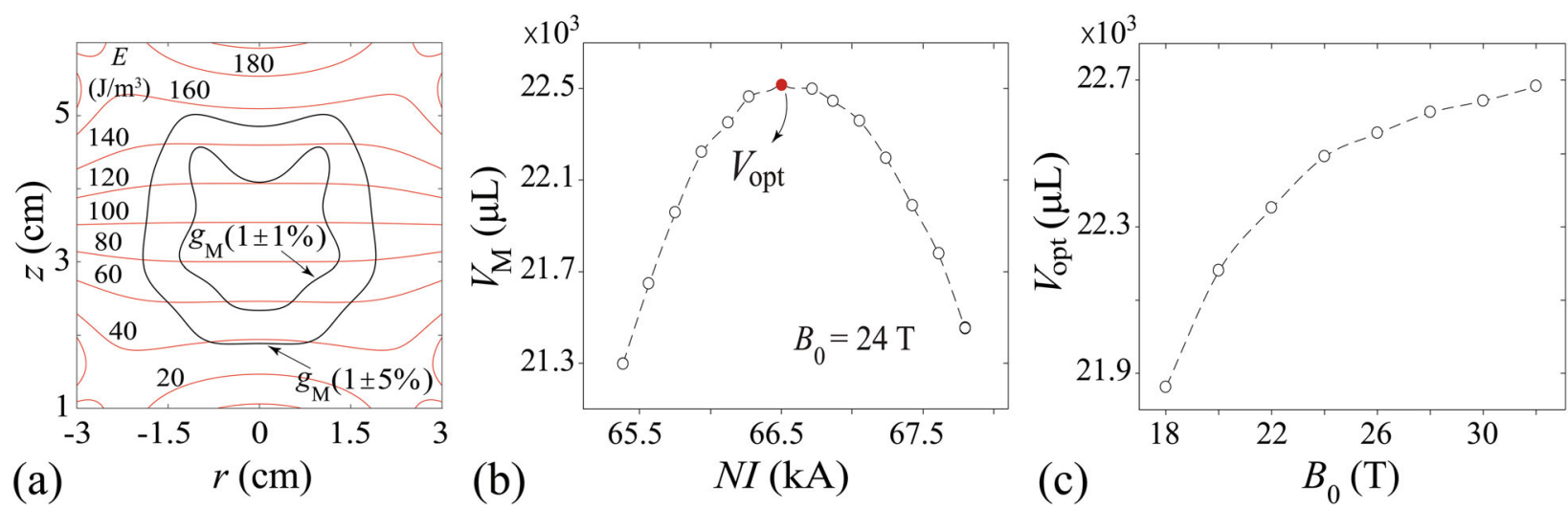

Fig. 6 Performance of the practical MLS as a Martian gravity simulator. a Contour plot of the specific potential energy $E(\mathbf{r})$ at $N I=66.55 \mathrm{kA}$ and $B_{0}=24 \mathrm{~T}$ in the practical MLS. The black contours denote the boundaries of the regions in which the total force leads to an effective gravitational acceleration within $1 \%$ and $5 \%$ of $g_{M}$, respectively. b The functional volume $V_{M}$ in which the gravity varies within $5 \%$ of $g_{M}$ versus the turn-current $N I$. c The peak volume $V_{\text {opt }}$ versus $B_{0}$.

This peak volume is so large that even small animals or plants can be accommodated inside. We have also calculated the peak volume $V_{\text {opt }}$ at different base field strength $B_{0}$. As shown in Fig. $6 c$, initially the peak volume $V_{\text {opt }}$ increases sharply with $B_{0}$, and then it gradually saturates when $B_{0}$ is $>\sim 24 \mathrm{~T}$. Operating the MLS at higher $B_{0}$ gives a marginal gain in the functional volume.

In conclusion, our analyses have clearly demonstrated the superiority of the proposed MSL concept in comparison with conventional solenoid MSLs. An unprecedentedly large and isotropic functional volume, i.e., about three orders of magnitude larger than that for a conventional solenoid MSL, can be achieved. The implementation of the superconducting magnet technology will also ensure the stable operation of this MLS with a minimal energy consumption rate, which is ideal for future low-gravity research and applications.

\section{METHOD}

\section{Magnetic field calculation}

The magnetic field $\mathbf{B}(\mathbf{r})$ generated at $\mathbf{r}$ by a current loop in threedimensional space can be calculated using the Biot-Savart law ${ }^{47}$ :

$\mathbf{B}(\mathbf{r})=\frac{\mu_{0} l}{4 \pi} \oint \frac{d \mathbf{I} \times(\mathbf{r}-\mathbf{I})}{|\mathbf{r}-\mathbf{I}|^{3}}$,

where $d \mathbf{l}$ is the elementary length vector along the current loop. For a field-gradient Maxwell coil with a radius $R=D / 2$, the generated magnetic field $\mathbf{B}_{1}(\mathbf{r})$ can be decomposed into an axial component and a radial component due to the axial symmetry. If we set the $z$ axis along the coaxial line of the two loops and place the coordinate origin at the center of the bottom loop, the two components can be evaluated as:

$$
\begin{aligned}
& B_{1}^{(r)}(r, z)=\frac{\mu_{0} l}{4 \pi} \int_{0}^{2 \pi}\left[\frac{R z \cos (\phi)}{R_{1}^{3}}+\frac{R(L-z) \cos (\phi)}{R_{2}^{3}}\right] d \phi \\
& B_{1}^{(z)}(r, z)=\frac{\mu_{0} l}{4 \pi} \int_{0}^{2 \pi}\left[\frac{R^{2}-R r \cos (\phi)}{R_{1}^{3}}+\frac{R r \cos (\phi)-R^{2}}{R_{2}^{3}}\right] d \phi
\end{aligned}
$$

where

$$
\begin{aligned}
& R_{1}=\sqrt{[r-R \cos (\phi)]^{2}+[R \sin (\phi)]^{2}+z^{2}} \\
& R_{2}=\sqrt{[r-R \cos (\phi)]^{2}+[R \sin (\phi)]^{2}+(z-L)^{2}},
\end{aligned}
$$

$L=\sqrt{3} D / 2$ is the separation distance between the two loops, and $l$ is the current in each loop.

The magnetic field $\mathbf{B}_{1}(\mathbf{r})$ generated by the practical MLS design as depicted in Fig. 5a can be calculated by superimposing the fields produced by the four sets of field-gradient Maxwell coils. The field of each coil is evaluated in the same way as outlined above. Counting in the base field $\mathbf{B}_{0}$, the total field is then given by $\mathbf{B}(\mathbf{r})=\left[B_{0}+B_{1}^{(z)}(\mathbf{r})\right] \hat{\mathbf{e}}_{z}+B_{1}^{(r)}(\mathbf{r}) \hat{\mathbf{e}}_{r}$

For a solenoid with a length $L$ and a radius $R$, if we assume the wire is thin such that the turn number $N$ is large but the total turn-current $N I$ remains finite, an exact expression for the generated magnetic field can be derived based on the Biot-Savart law ${ }^{63,64}$ :

$$
\begin{aligned}
& B^{(r)}(r, z)=\frac{\mu_{0} N I I}{4 \pi} \frac{2}{L} \sqrt{\frac{R}{r}}\left[\frac{k^{2}-2}{k} K\left(k^{2}\right)+\frac{2}{k} E\left(k^{2}\right)\right]_{\zeta_{-}}^{\zeta_{+}} \\
& B^{(z)}(r, z)=\frac{\mu_{0} N I}{4 \pi} \frac{1}{L \sqrt{R r}}\left[\zeta k\left(K\left(k^{2}\right)+\frac{R-r}{R+r} \Pi\left(h^{2}, k^{2}\right)\right)\right]_{\zeta_{-}}^{\zeta_{+}}
\end{aligned}
$$

where

$$
\begin{aligned}
k^{2} & =\frac{4 R r}{(R+r)^{2}+\zeta^{2}} \\
h^{2} & =\frac{4 R r}{(R+r)^{2}} \\
\zeta_{ \pm} & =z \pm L / 2,
\end{aligned}
$$

and the functions $K\left(k^{2}\right), E\left(k^{2}\right)$, and $\Pi\left(h^{2}, k^{2}\right)$ are given by:

$$
\begin{aligned}
K\left(k^{2}\right) & =\int_{0}^{\pi / 2} \frac{d \theta}{\sqrt{1-k^{2} \sin ^{2} \theta}} \\
E\left(k^{2}\right) & =\int_{0}^{\pi / 2} d \theta \sqrt{1-k^{2} \sin ^{2} \theta} \\
\Pi\left(h^{2}, k^{2}\right) & =\int_{0}^{\pi / 2} \frac{d \theta}{\left(1-h^{2} \sin ^{2} \theta\right) \sqrt{1-k^{2} \sin ^{2} \theta}} .
\end{aligned}
$$

\section{Numerical method}

The magnetic fields produced by the solenoid, the ideal gradient-field Maxwell coil, and the practical MLS design are all calculated using MATLAB. Considering the axial symmetry, we only evaluate the fields in the $r-z$ plane. The sizes of the computational domains for different types of designs are essentially shown in Fig. 1b, Fig. 2b, and Fig. 5b. Typically, the computational domain is discretized using a square grid with spatial resolutions $\Delta r=10 \mu \mathrm{m}$ and $\Delta z=10 \mu \mathrm{m}$, which gives good convergence of the numerical results. The calculations assumed water properties at the ambient temperature, but the same procedures can be applied to other materials with different magnetic susceptibilities and densities.

\section{Reporting summary}

Further information on research design is available in the Nature Research Reporting Summary linked to this article.

\section{DATA AVAILABILITY}

The computer codes and the data supporting the findings of this study are available from the corresponding author upon reasonable request.

Received: 1 August 2021; Accepted: 8 October 2021; Published online: 29 October 2021

\section{REFERENCES}

1. Hammond, T. G. et al. Gene expression in space. Nat. Med. 5, 359-359 (1999). 2. White, R. J. \& Averner, M. Humans in space. Nature 409, 1115-1118 (2001). 
3. Williams, D., Kuipers, A., Mukai, C. \& Thirsk, R. Acclimation during space flight: effects on human physiology. Can. Med. Assoc. J. 180, 1317-1323 (2009).

4. Stavnichuk, M., Mikolajewicz, N., Corlett, T., Morris, M. \& Komarova, S. V. A systematic review and meta-analysis of bone loss in space travelers. npj Microgravity 6, 1-9 (2020).

5. Snyder, H. A. Effect of sloshing on the mechanics of dewar systems in low-gravity. Cryogenics 41, 825-832 (2001).

6. Nelson, A. R. \& Gokhale, N. R. Oscillation frequencies of freely suspended water drops. J. Geophys. Res. 77, 2724-2727 (1972).

7. Obreschkow, D. et al. Cavitation bubble dynamics inside liquid drops in microgravity. Phys. Rev. Lett. 97, 094502 (2006).

8. Siegel, R. Effects of reduced gravity on heat transfer. In Adv. Heat Transf. 4, 143-228 (1967).

9. Ohta, H. \& Baba, S. Boiling experiments under microgravity conditions. Exp. Heat. Transf. 26, 266-295 (2013)

10. Unsworth, B. R. \& Lelkes, P. I. Growing tissues in microgravity. Nat. Med. 4, 901-907 (1998)

11. Strelov, V. I., Kuranova, I. P., Zakharov, B. G. \& Voloshin, A. E. Crystallization in space: results and prospects. Crystallogr. Rep. 59, 781-806 (2014).

12. Matisak, B. P., Zhao, A. X., Narayanan, R. \& Fripp, A. L. The microgravity environment: its prediction, measurement, and importance to materials processing. $J$. Cryst. Growth 174, 90-95 (1997).

13. Board, S.S. \& National Research Council of The National Academia. Assessment of NASA's Mars Architecture 2007-2016. The National Academies Press (2006)

14. Witze, A. NASA plans Mars sample-return rover. Nature 509, 272 (2014).

15. Musk, E. Making life multi-planetary. N. Space 6, 2-11 (2018).

16. Vandenbrink, J. P. \& Kiss, J. Z. Space, the final frontier: A critical review of recent experiments performed in microgravity. Plant Sci. 243, 115-119 (2016).

17. Penley, N. J., Schafer, C. P. \& Bartoe, J. F. The international space station as a microgravity research platform. Acta Astronaut. 50, 691-696 (2002).

18. Ferranti, F., Del Bianco, M. \& Pacelli, C. Advantages and limitations of current microgravity platforms for space biology research. Appl. Sci. 11, 68 (2021).

19. Selig, H., Dittus, H. \& Lämmerzahl, C. Drop tower microgravity improvement towards the nano- $g$ level for the MICROSCOPE payload tests. Microgravity Sci. Technol. 22, 539-549 (2010).

20. Liu, T. Y., Wu, Q. P., Sun, B. Q. \& Han, F. T. Microgravity level measurement of the Beijing drop tower using a sensitive accelerometer. Sci. Rep. 6, 1-9 (2016).

21. Melnikov, D. E., Ryzhkov, I. I., Mialdun, A. \& Shevtsova, V. Thermovibrational convection in microgravity: preparation of a parabolic flight experiment. Microgravity Sci. Technol. 20, 29-39 (2008).

22. Shelhamer, M. Parabolic flight as a spaceflight analog. J. Appl. Physiol. 120, 1442-1448 (2016).

23. Fuhrmann, E. \& Dreyer, M. Description of the sounding rocket experiment SOURCE. Microgravity Sci. Technol. 20, 205-212 (2008).

24. Ohta, $H$. et al. TR-1A rocket experiment on nucleate pool boiling heat transfer under microgravity. ASME J. Dyn. Syst. Meas. Control 62, 249-256 (1997).

25. Sundaresan, A., Risin, D., \& Pellis, N. R. Cell growth in microgravity. In Reviews in Cell Biology and Molecular Medicine. American Cancer Society (2006).

26. Pletser, V. Short duration microgravity experiments in physical and life sciences during parabolic flights: the first 30 ESA campaigns. Acta Astronaut. 55, 829-854 (2004).

27. Dedolph, R. R. \& Dipert, M. H. The physical basis of gravity stimulus nullification by clinostat rotation. Plant Physiol. 47, 756-764 (1971).

28. Herranz, R. et al. Ground-based facilities for simulation of microgravity: organismspecific recommendations for their use, and recommended terminology. Astrobiology 13, 1-17 (2013).

29. Carlsson, S. I., Bertilaccio, M. T., Ballabio, E. \& Maier, J. A. Endothelial stress by gravitational unloading: effects on cell growth and cytoskeletal organization. Biochim. Biophys. Acta Mol. Cell Res. 1642, 173-179 (2003).

30. Wuest, S. L., Richard, S., Kopp, S., Grimm, D. \& Egli, M. Simulated microgravity: critical review on the use of random positioning machines for mammalian cell culture. BioMed. Res. Int. 2015, 1-8 (2015).

31. Hoson, T., Kamisaka, S., Masuda, Y., Yamashita, M. \& Buchen, B. Evaluation of the three-dimensional clinostat as a simulator of weightlessness. Planta 203, S187-S197 (1997)

32. Brown, A. H., Dahl, A. O. \& Chapman, D. K. Limitation on the use of the horizontal clinostat as a gravity compensator. Plant Physiol. 58, 127-130 (1976).

33. Albrecht-Buehler, G. The simulation of microgravity conditions on the ground. Gravit. Space Res. 5, 3-10 (2007).

34. Hill, R. J. \& Eaves, L. Nonaxisymmetric shapes of a magnetically levitated and spinning water droplet. Phys. Rev. Lett. 101, 234501 (2008).

35. Brooks, J. S. et al. New opportunities in science, materials, and biological systems in the low-gravity (magnetic levitation) environment. J. Appl. Phys. 87, 6194-6199 (2000).

36. Weilert, M. A., Whitaker, D. L., Maris, H. J. \& Seidel, G. M. Magnetic levitation and noncoalescence of liquid helium. Phys. Rev. Lett. 77, 4840 (1996).
37. Berry, M. V. \& Geim, A. K. Of flying frogs and levitrons. Eur. J. Phys. 18, 307 (1997).

38. Valles Jr, J. M., Lin, K., Denegre, J. \& Mowry, K. Stable magnetic field gradient levitation of Xenopus laevis: toward low-gravity simulation. Biophys. J. 73, 1130-1133 (1997).

39. Liu, Y., Zhu, D., Strayer, D. M. \& Israelsson, U. Magnetic levitation of large water droplets and mice. Adv. Space Res. 45, 208-213 (2010).

40. Geim, A. K. Everyone's magnetism. Phys. Today 51, 36-39 (1998)

41. Schenck, J. F. Safety of strong, static magnetic fields. J. Magn. Reson. Imaging 12, 2-19 (2000)

42. High, W. B., Sikora, J., Ugurbil, K. \& Garwood, M. Subchronic in vivo effects of a high static magnetic field (9.4 Tesla) in rats. J. Magn. Reson. Imaging 12, 122-139 (2000).

43. Valles Jr, J. M., Maris, H. J., Seidel, G. M., Tang, J. \& Yao, W. Magnetic levitationbased Martian and Lunar gravity simulator. Adv. Space Res. 36, 114-118 (2005).

44. Chow, T., Wong, P. L. \& Liu, K. P. Shape effect of magnetic source on stabilizing range of vertical diamagnetic levitation. IEEE Trans. Magn. 48, 26-30 (2011).

45. Kustler, G., Nemoianu, I. V. \& Cazacu, E. Theoretical and experimental investigation of multiple horizontal diamagnetically stabilized levitation with permanent magnets. IEEE Trans. Magn. 48, 4793-4801 (2012).

46. Kuznetsov, S. \& Guest, J. K. Topology optimization of magnetic source distributions for diamagnetic and superconducting levitation. J. Magn. Magn. Mater. 438, 60-69 (2017).

47. Jackson, J. D. Classical Electrodynamics. American Association of Physics Teachers, 3rd ed (1999).

48. Konishi, C. \& Mudawar, I. Review of flow boiling and critical heat flux in microgravity. Int. J. Heat Mass Transf. 80, 469-493 (2015).

49. Straub, J. Boiling heat transfer and bubble dynamics in microgravity. In Adv. Heat Transf. 35, 57-172 (2001).

50. Tegart, J. R. \& Butz, J. R. Analysis of Skylab fluid mechanics science demonstrations. Martin Marietta Corporation, Prepared for National Aeronautics and Space Administration (1975).

51. Cooper, G. M., Hausman, R. E., \& Hausman, R. E. The Cell: A molecular approach, Vol. 4, ASM press Washington, DC, 4th ed (2007).

52. Eisenberg, D., Kauzmann, W., \& Kauzmann, W. The Structure and Properties of Water. Oxford University Press on Demand (2005).

53. Van Sciver, S. W. \& Marken, K. R. Superconducting magnets above 20 Tesla. Phys. Today 55, 37-42 (2002).

54. Dixon, I. R., Markiewicz, W. D., Brey, W. W. \& Shetty, K. K. Performance of the ultra wide bore $900 \mathrm{MHz}$ NMR magnet at the national high magnetic field laboratory. IEEE Trans. Appl. Supercond. 15, 1334-1337 (2005).

55. Moser, E., Laistler, E., Schmitt, F. \& Kontaxis, G. Ultra-high field NMR and MRI- the role of magnet technology to increase sensitivity and specificity. Front. Phys. 5, 33 (2017).

56. Markiewicz, W. D. et al. Design of a superconducting 32-T magnet with REBCO high field coils. IEEE Trans. Appl. Supercond. 22, 4300704-4300704 (2011).

57. Maxwell, J. C. A Treatise on Electricity and Magnetism, Vol. 1, Clarendon Press (1873).

58. Fichtl, G. H., Theon, J. S., Hil, C. K., \& Otha, H. Spacelab 3 Mission science review. NASA conference publication. Scientific and Technical Information Branch (1987).

59. Hahn, S. et al. 45.5-tesla direct-current magnetic field generated with a hightemperature superconducting magnet. Nature 570, 496-499 (2019).

60. Fu, R. et al. Ultra-wide bore $900 \mathrm{MHz}$ high-resolution NMR at the national high magnetic field laboratory. J. Magn. Reson. Imaging 177, 1-8 (2005).

61. Hendrickson, C. et al. 21 Tesla Fourier transform ion cyclotron resonance mass spectrometer: a national resource for ultrahigh resolution mass analysis. J. Am. Soc. Mass Spectrom. 26, 1626-1632 (2015).

62. Braccini, V. et al. Properties of recent IBAD-MOCVD coated conductors relevant to their high field, low temperature magnet use. Supercond. Sci. Technol. 24, 035001 (2010).

63. Callaghan, E. E., \& Maslen, S. H. The magnetic field of a finite solenoid, Vol. 465, National Aeronautics and Space Administration Washington (1960).

64. Caciagli, A., Baars, R. J., Philipse, A. P. \& Kuipers, B. W. Exact expression for the magnetic field of a finite cylinder with arbitrary uniform magnetization. J. Magn. Magn. Mater. 456, 423-432 (2018).

\section{ACKNOWLEDGMENTS}

The authors would like to thank H.J. Maris and J. Valles for their valuable comments on the manuscript. The authors also acknowledge Shaolong Zhu for his assistance at the early stage. This research is supported by National Science Foundation under grant no. CBET-1801780. The work was conducted at the National High Magnetic Field Laboratory, Florida State University, which is supported by National Science Foundation Cooperative Agreement No. DMR-1644779 and the state of Florida. 


\section{AUTHOR CONTRIBUTIONS}

W.G. designed the research; H.S. conducted the simulations; H.S. and W.G. analyzed the results and wrote the paper.

\section{COMPETING INTERESTS}

The authors declare no competing interests.

\section{ADDITIONAL INFORMATION}

Supplementary information The online version contains supplementary material available at https://doi.org/10.1038/s41526-021-00174-4.

Correspondence and requests for materials should be addressed to Wei Guo.

Reprints and permission information is available at http://www.nature.com/ reprints
Publisher's note Springer Nature remains neutral with regard to jurisdictional claims in published maps and institutional affiliations.

(i) Open Access This article is licensed under a Creative Commons Attribution 4.0 International License, which permits use, sharing, adaptation, distribution and reproduction in any medium or format, as long as you give appropriate credit to the original author(s) and the source, provide a link to the Creative Commons license, and indicate if changes were made. The images or other third party material in this article are included in the article's Creative Commons license, unless indicated otherwise in a credit line to the material. If material is not included in the article's Creative Commons license and your intended use is not permitted by statutory regulation or exceeds the permitted use, you will need to obtain permission directly from the copyright holder. To view a copy of this license, visit http://creativecommons. org/licenses/by/4.0/.

(c) The Author(s) 2021 